\section{Selective case finding \\ versus universal screening \\ for detecting hypothyroidism \\ in the first trimester of pregnancy: \\ a comparative evaluation of \\ a group of pregnant women \\ from Rio de Janeiro}

Tatiana Martins Benvenuto Louro Berbara'

https://orcid.org/0000-0003-3435-351X

Nathalie Silva de Morais ${ }^{1}$

https://orcid. org/0000-0002-7883-8175

Débora Ayres Saraiva ${ }^{1}$

https://orcid.org/0000-0001-8494-0435

Carolina Martins Corcino ${ }^{1}$

https://orcid. org/0000-0003-3111-9736

Annie Schtscherbyna ${ }^{1}$

https://orcid. org/0000-0002-6403-0036

Karina Lúcia Moreira ${ }^{1}$

https://orcid.org/0000-0002-4269-4966

Patrícia de Fátima dos Santos Teixeira ${ }^{1}$

https://orcid. org/0000-0001-8859-6387

Mario Vaisman ${ }^{1}$

https://orcid.org/0000-0002-9469-4298

\begin{abstract}
Objective: Maternal hypothyroidism during pregnancy may lead to adverse outcomes. Recently published guidelines by the American Thyroid Association (ATA) do not advocate for universal screening but recommend a case-finding approach in high-risk pregnant women. The present study aims to evaluate the accuracy of this approach in identifying women with thyroid dysfunction during early pregnancy. Subjects and methods: This is a multiple-center, cross-sectional study. Three hundred and one pregnant women were enrolled. Anamnesis and a physical examination were performed to detect which women fulfilled the criteria to undergo laboratory screening of thyroid dysfunction, according to the ATA's 2017 guidelines. The Zulewski's validated clinical score was applied to assess signs and symptoms of hypothyroidism. Serum levels of thyrotropin (TSH), free thyroxine (FT4), anti-thyroperoxidase (TPO-Ab), and anti-thyroglobulin (Tg-Ab) antibodies were determined. Results: Two hundred and thirty one women (78\%) were classified as high risk, and 65 (22\%) were classified as low risk for thyroid dysfunction. Regarding the clinical score, 75 patients (31.2\%) presented mild symptoms that were compatible with $\mathrm{SCH}$, of which $22(7.4 \%)$ had symptoms as the only risk factor for thyroid disease. 17 patients $(5.7 \%)$ had $\mathrm{SCH}$, of which $10(58.8 \%)$ belonged to the high-risk group, and $7(41.2 \%)$ belonged to the low-risk group. $\mathrm{OH}$ was found in 4 patients $(1.4 \%)$ : $3(75 \%)$ in the high-risk group and $1(25 \%)$ in the low-risk group. Conclusions: The ATA's proposed screening criteria were not accurate in the diagnosis of thyroid dysfunction in pregnancy. Testing only the high-risk pregnant women would miss approximately $40 \%$ of all hypothyroid patients. Arch Endocrinol Metab. 2020;64(2):159-64
\end{abstract}

Keywords
1 Departamento de Medicina Interna, Unidade de Endocrinologia, Faculdade de Medicina, Hospital Universitário Clementino Fraga Filho, Universidade Federal do Rio de Janeiro (HUCFF/UFRJ), Rio de Janeiro, RJ, Brasil
Correspondence to:

Tatiana Martins Benvenuto Louro Berbara

Hospital Universitário

Clementino Fraga Filho

Serviço de Endocrinologia

Rua Rodolpho Paulo Rocco, 255

$9^{\circ}$ andar, sala $9 \mathrm{E} 23$

21941-913 - Rio de Janeiro, RJ, Brasil

tatiberbara.med@gmail.com

Received on May/19/2018 Accepted on Sept/13/2019

DOI: 10.20945/2359-3997000000209 
Hypothyroidism; pregnancy; universal screening; case-finding

\section{INTRODUCTION} $\mathbf{M} \begin{aligned} & \text { aternal hypothyroidism, especially when } \\ & \text { diagnosed during the first trimester of gestation, }\end{aligned}$ may lead to adverse obstetric and neonatal outcomes as well as impaired neurocognitive development of the fetus (1-4). Recently published guidelines by the American Thyroid Association (ATA) do not advocate for universal screening but recommend case finding in high-risk pregnant women (5). According to this recommendation, all patients should undergo clinical evaluation during the first prenatal visit in order to identify the presence of risk factors for thyroid disease. If any of the risk factors, which were reviewed in the recent guidelines, are identified, testing for serum TSH during the first trimester of gestation is recommended (5).

Previously, the European guidelines did not advocate for universal screening because of the lack of grade 1 evidence, but they noted that the majority of the authors recommended it, considering the beneficial effects of levothyroxine (LT4) treatment on unknown overt hypothyroidism $(\mathrm{OH})$ and the fact that the targeted approach will miss a large percentage of women with subclinical hypothyroidism (SCH) (6). Indeed, several studies have reported that case-based screening may result in failure to detect a large number of low-risk pregnant women with thyroid disease (7-9).

Besides the evidence that the case-finding approach may not be effective, universal thyroid screening during early pregnancy fulfils most criteria for a beneficial and cost-effective screening programme, according to recommendations of the WHO (10). Thyroid disease is an important health problem in pregnancy, which can be easily diagnosed with non-invasive and inexpensive diagnostic tests, and has a safe and welltolerated treatment available. Screening all pregnant women has shown to be cost effective, even if only $\mathrm{OH}$ was considered to be associated with adverse obstetric outcomes (11).

The normal reference range for serum TSH concentrations in pregnancy, as well as the recommendations for LT4 treatment were also updated in the recent ATA guidelines. They reinforced the need to establish population-based trimester-specific reference ranges for serum TSH. When not available, the upper reference limit during the first trimester of pregnancy can be defined by reducing $0.5 \mathrm{mIU} / \mathrm{L}$ from the upper limit of TSH for non-pregnant women (5).
In Brazil, controversy regarding the best screening strategy of thyroid disease in pregnant women also exists. Therefore, we designed the present study to evaluate the accuracy of the ATA's high-risk casefinding approach in identifying women with thyroid dysfunction during early pregnancy.

\section{SUBJECTS AND METHODS}

This multiple-center, cross-sectional study was performed with an ongoing prospective cohort that included pregnant women attending prenatal programs in four public health care units from an urban area with iodine sufficiency (12) in a Brazilian coastal state. During the recruitment period, each unit was visited regularly, but at different times - not necessarily concomitantly. The average time of visit to each center was 10 to 12 months. All pregnant women who attended the units during our visit and met the study inclusion criteria were enrolled.

A total of 301 pregnant women attending at their first prenatal visit were included from September 2014 to January 2018. The recruiting criteria included age $\geq 18$ years old, having a spontaneous pregnancy, and gestational age up to 12 weeks (defined by last menstrual period or ultrasound). Exclusion criteria were multifetal pregnancy, known autoimmune thyroid disease, or current use of levothyroxine, antithyroid drug, or nutritional supplements containing iodine. All subjects provided informed written consent, and the study was approved by the local research ethics committee (CAAE: 22546213.0.0000.5275).

From the initial sample of 301 pregnant women, 3 were excluded because they had a gestational age greater than 12 weeks, 1 was excluded because she was in treatment for hypothyroidism, and 1 was excluded because of a twin pregnancy. The final sample included 296 pregnant women.

Anamnesis and a general physical examination were performed to detect which pregnant women fulfilled the criteria for laboratory screening of thyroid dysfunction, according to 2017 ATA guidelines (5). The screening criteria are shown in table l. If any of the criteria were identified, the patient was considered to be at high risk for thyroid dysfunction. Patients who did not meet any criteria were classified as low risk for thyroid dysfunction. The Zulewski's validated clinical score was applied to assess signs and symptoms of hypothyroidism (13). According to this scoring system, women were considered clinically hypothyroid when they achieved 
Table 1. Risk factors for thyroid dysfunction during pregnancy, according to ATA's 2017 guidelines

1. A history of hypothyroidism/hyperthyroidism or current symptoms/signs of
thyroid dysfunction
2. Known thyroid antibody positivity or presence of a goiter
3. History of head or neck radiation or prior thyroid surgery
4. Age $>30$ years
5. Type 1 diabetes or other autoimmune disorders
6. History of pregnancy loss, preterm delivery, or infertility
7. Multiple prior pregnancies $(\geq 2)$
8. Family history of autoimmune thyroid disease or thyroid dysfunction
9. Morbid obesity (BMl $\geq 40 \mathrm{~kg} / \mathrm{m}^{2}$ )
10. Use of amiodarone or lithium, or recent administration of iodinated
radiologic contrast
11. Residing in an area of known moderate to severe iodine insufficiency
a American Thyroid Association.

more than 5 points; euthyroid with 0-2 points; and subclinically hypothyroid with 3-5 points (13). Body mass index (BMI) was calculated as weight $(\mathrm{kg})$ divided by height squared $\left(\mathrm{m}^{2}\right)$. As previously reported, the population studied was considered to have iodine sufficiency (12).

Morning blood samples were collected from all patients to determine serum levels of thyrotropin (TSH), free thyroxine (FT4), anti-thyroperoxidase (TPO-Ab), and anti-thyroglobulin ( $\mathrm{Tg}-\mathrm{Ab})$ antibodies. Diagnosis of $\mathrm{OH}$ required a serum TSH $>10.0 \mathrm{mIU} / \mathrm{L}$ or FT4 below the inferior reference range (associated with serum TSH elevations). Isolated hypothyroxinemia was defined by FT4 below the inferior range with normal TSH. TSH elevations above $3.8 \mathrm{mIU} / \mathrm{L}$ (and $<10.0 \mathrm{mIU} / \mathrm{L}$ ) with normal FT4 constituted a diagnosis of $\mathrm{SCH}$. We considered $3.8 \mathrm{mIU} / \mathrm{L}$ as the upper reference limit, by reducing 0.5 from the upper limit of TSH for non-pregnant women, according to recent recommendations from ATA's guidelines (5). This value is similar to that found by Silva de Morais and cols.., who evaluated the TSH reference range in a group of pregnant women from Rio de Janeiro (14).

Serum TSH, FT4, TPO-Ab, and Tg-Ab were determined by an electrochemiluminescence immunometric assay with a Roche Modular Analytics ${ }^{\circledR}$ E170 (Roche Diagnostics). The laboratory reference ranges of TSH were 0.4 to $4.3 \mathrm{mIU} / \mathrm{L}$ (for nonpregnant women), FT4 0.7 to $1.9 \mathrm{ng} / \mathrm{dL}, \mathrm{TPO}-\mathrm{Ab}<34 \mathrm{IU} / \mathrm{mL}$, and $\mathrm{Tg}-\mathrm{Ab}<115 \mathrm{IU} / \mathrm{mL}$. The intra- and interassay coefficients of variation of serum TSH, FT4, TPO-Ab, and $\mathrm{Tg}-\mathrm{Ab}$ were, respectively, $7.2 \%$ and $3 \%, 2.8 \%$ and $2.9 \%, 6.3 \%$ and $7.0 \%$, and $4.9 \%$ and $6.3 \%$.

The associations between the risk classification and the occurrence of thyroid dysfunction and autoimmunity were evaluated by the Chi-square test. The observed p-values were obtained by Monte Carlo simulations (15) and corrected by the Sidàk procedure to multiple tests (16). In addition to the association analysis, we evaluated the sensitivity, specificity, positive and negative predictive values, and the accuracy of the ATA's criteria for laboratory screening of thyroid dysfunction (5).

\section{RESULTS}

We evaluated thyroid function and thyroid autoimmunity in 296 pregnant women. The characteristics of these women are shown in table 2 .

Regarding the clinical score, 75 patients (31.2\%) presented mild symptoms that were compatible with SCH, of which $22(7.4 \%)$ had symptoms as the only risk factor for thyroid disease. Since no women had symptoms consistent with $\mathrm{OH}$, we considered the presence of mild symptoms as a positive criteria for screening for thyroid dysfunction.

According to the criteria established by the 2017 ATA guidelines (5), 231 women (78\%) were classified as high risk, and $65(22 \%)$ were classified as low risk for thyroid dysfunction during pregnancy. In previous recommendations, $\geq 2$ prior pregnancies were not considered one of the defining criteria of the high-risk

Table 2. Baseline characteristics of the 296 pregnant women enrolled in the study:

\begin{tabular}{|c|c|}
\hline Mean $( \pm$ SD) maternal age (years) & $28.8( \pm 5.9)$ \\
\hline Mean ( \pm SD) gestational age at screening (weeks) & $9.1( \pm 2)$ \\
\hline$\geq 2$ prior pregnancies, $\mathrm{n}(\%)$ & $76(25.7)$ \\
\hline History of previous miscarriages, n (\%) & $55(19.1)$ \\
\hline History of fetal death and/or preterm delivery, $\mathrm{n}(\%)$ & $10(3.4)$ \\
\hline History of infertility, $n$ (\%) & $32(11.9)$ \\
\hline Family history of thyroid disease, $n(\%)$ & $43(14.3)$ \\
\hline Personal history of other autoimmune diseases, $n$ (\%) & $2(0.7)$ \\
\hline History of head/neck irradiation and/or thyroid surgery, $n$ (\%) & $1(0.4)$ \\
\hline$B M l^{\mathrm{a}} \geq 40, \mathrm{n}(\%)$ & $3(1)$ \\
\hline $\begin{array}{l}\text { Clinical euthyroidism ( } 0-2 \text { points in the Zulewski's clinical } \\
\text { score), } n(\%)\end{array}$ & $165(68.8)$ \\
\hline $\begin{array}{l}\text { Signs and symptoms of } \mathrm{SCH}^{\mathrm{b}}(3-5 \text { points in the Zulewski's } \\
\text { clinical score), } \mathrm{n}(\%)\end{array}$ & $75(31.2)$ \\
\hline $\begin{array}{l}\text { Signs and symptoms of } \mathrm{OH}^{c}(>5 \text { points in the Zulewski's } \\
\text { clinical score), } \mathrm{n}(\%)\end{array}$ & $0(0)$ \\
\hline
\end{tabular}


group (17). According to the most recent guidelines, 10 women $(3.4 \%)$ were newly classified as high risk for thyroid dysfunction, based on multiparity.

Regarding thyroid function, the median TSH was $1.42 \mathrm{mIU} / \mathrm{L}$. SCH was found in 17 patients $(5.7 \%)$, and $\mathrm{OH}$ was found in 4 patients (1.4\%). The prevalence of thyroid dysfunction according to risk classification is shown in figure 1 .

A total of 17 women (5.7\%) were positive for TPO$\mathrm{Ab}$, and 17 of $276(6.2 \%)$ were positive for $\mathrm{Tg}-\mathrm{Ab} .7$ patients $(2.54 \%)$ were positive for both TPO-Ab and Tg-Ab. 20 (6.8\%) had no record of $\mathrm{Tg}$-Ab values.

The associations between risk classification and occurrence of thyroid dysfunction, as well as the sensitivity, specificity, positive and negative predictive values, and accuracy of the ATA's risk criteria are shown in table 3 .

Of 17 patients with SCH, $10(58.8 \%)$ belonged to the high-risk group, and 7 (41.2\%) belonged to the lowrisk group. Sensitivity, specificity, positive predictive value (PPV), negative predictive value (NPV), and

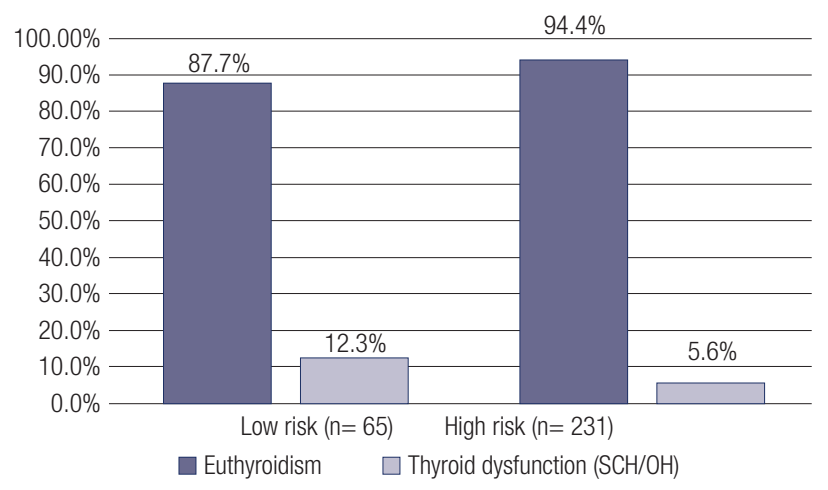

Figure 1. Prevalence of thyroid dysfunction according to risk classification accuracy of the screening criteria were $58.8 \%, 20.8 \%$, $4.3 \%, 89.2 \%$, and $23 \%$, respectively.

The criteria were also not sensitive $(75 \%)$ or specific (21.9\%) for the diagnosis of $\mathrm{OH}$.

Additionally, the associations between risk classification and the presence of autoimmunity are shown in table 4.

No statistically significant association was observed between risk classification and thyroid dysfunction or presence of autoimmunity. A post-hoc analysis showed that the sample had a statistical power of $82 \%$ to detect small effect sizes $(0.2)$ and $99.9 \%$ for medium effect sizes (0.5), according to Cohen's criteria (18).

In its 2017 guidelines (5), the ATA suggests, based on moderate quality of evidence, that treatment with levothyroxine should be considered for pregnant women with $\mathrm{TSH}>2.5 \mathrm{mIU} / \mathrm{L}$, associated with the presence of autoantibodies. In our cohort, we found that 8 patients $(2.7 \%)$ would receive thyroid hormone replacement according to this criteria: 4 patients in the high-risk group and 4 in the low-risk group.

\section{DISCUSSION}

This multiple-center, cross-sectional study shows that the criteria proposed by the ATA's 2017 guidelines do not represent a good screening tool for hypothyroidism in pregnancy. This was demonstrated by the absence of a significant association between risk classification and occurrence of $\mathrm{SCH}$. In addition, the sensitivity found was below $60 \%$ and the specificity was around $20 \%$. More important than these results are those of predictive values. The positive predictive value (PPV) was below $5 \%$. Thus, when determining that a pregnant woman is at high risk, the probability of SCH is less than $5 \%$. This result is of very limited utility in clinical practice. The negative

Table 3. Frequency distribution of individuals with thyroid dysfunction according to the risk classification, and the sensitivity, specificity, PPV', NPV', accuracy, and $p$-value

\begin{tabular}{lcccccccc}
\hline & High risk & Low risk & p-value & Sensitivity & Specificity & PPV $^{\mathbf{a}}$ & NPV $^{\mathbf{b}}$ & Accuracy \\
\hline $\mathrm{SCH}^{\mathrm{c}}$ & $10(58.8 \%)$ & $7(41.2 \%)$ & 0.232 & 58.8 & 20.8 & 4.3 & 89.2 & 23 \\
$\mathrm{OH}^{\mathrm{d}}$ & $3(75 \%)$ & $1(25 \%)$ & 0.999 & 75 & 21.9 & 1.3 & 98.5 & 22.6 \\
\hline
\end{tabular}

${ }^{a}$ positive predictive value; ${ }^{\mathrm{b}}$ negative predictive value; ${ }^{\mathrm{c}}$ subclinical hypothyroidism; ${ }^{\mathrm{d}}$ overt hypothyroidism.

Table 4. Frequency distribution of individuals with autoantibodies according to the risk classification, and the sensitivity, specificity, PPVª NPV $^{\mathrm{b}}$, accuracy, and $p$-value

\begin{tabular}{|c|c|c|c|c|c|c|c|c|}
\hline & High risk & Low risk & p-value & Sensitivity & Specificity & $P P V^{a}$ & $N^{\prime} V^{b}$ & Accuracy \\
\hline TPO-Abc positive & $13(76.5 \%)$ & $4(23.5 \%)$ & 0.997 & 76.5 & 21.9 & 5.6 & 93.9 & 25 \\
\hline $\mathrm{Tg}-\mathrm{Ab} \mathrm{b}^{\mathrm{b}}$ positive & 14 (82.4\%) & $3(17.6 \%)$ & 0.999 & 82.4 & 22.4 & 6.5 & 95.1 & 26.1 \\
\hline
\end{tabular}

a positive predictive value; ${ }^{\mathrm{b}}$ negative predictive value; ${ }^{\mathrm{c}}$ anti-thyroperoxidase; ${ }^{\mathrm{d}}$ anti-thyroglobulin. 
predictive value (NPV), in turn, increased to around 90\%, indicating that pregnant women in the low-risk group still presented a $10 \%$ probability of developing $\mathrm{SCH}$.

Finally, we observed a low accuracy, showing a weak predictive capacity of the screening criteria in the diagnosis of $\mathrm{SCH}$.

Regarding $\mathrm{OH}$, there was also no significant association between risk and occurrence of overt thyroid dysfunction. The sensitivity, specificity, and PPV were $75 \%, 21.92 \%$, and $1.3 \%$, respectively. We found a high NPV (98.5\%), but this result should be interpreted with caution, since it is probably due to the very low number of $\mathrm{OH}$ cases.

If the targeted high-risk case-finding approach is used, 7 women (41.2\%) with SCH, and 1 woman $(25 \%)$ with $\mathrm{OH}$ would be missed, since they belonged to the low-risk group. 4 women $(50 \%)$ with positive autoantibodies and TSH $>2.5 \mathrm{mIU} / \mathrm{L}$, for whom treatment with LT4 would be considered according to the new recommendations (5), would also be missed.

Rosario and cols. who evaluated 412 low-risk pregnant women in a metropolitan region of Belo Horizonte (Minas Gerais, Brazil) found that selective screening, recommended by ATA, does not result in a significant loss of women with an indication for LT4 treatment (19). These results differs from ours, possibly due to differences in patient selection, since it is unclear how assessment of signs and symptoms of hypothyroidism was made. In our study, 75 women (31.2\%) had mild symptoms, according to Zulewski's clinical score, of which $22(7.4 \%)$ had symptoms as the only risk factor for thyroid disease. Considering that Brazil is a country of continental proportions, it is also possible that the divergences found are due to demographic differences, such as different profiles of iodine consumption. Our population was considered to have iodine sufficiency (12).

On the other hand, our results are similar to a number of other studies. Vaidya and cols., evaluated a cohort of 1560 pregnant women (7). Of all women with elevated TSH, 30\% belonged to the low-risk group. In a retrospective cohort study performed in 2011 with data from the United States, Chang and cols. found that among 983 pregnant women in Boston, Massachusetts, up to $80 \%$ of women with elevated TSH levels might have been missed using a case-finding approach rather than universal screening (8). In 2015, Jouyandeh and cols. performed a systematic review and meta-analysis to compare the efficacy of universal screening versus a high-risk case-finding approach (9). Ten eligible articles were selected, and the results showed that the overall loss ratio in case-finding method was $49 \%$ (CI $0.23-0.74$ ).

Another important issue to be considered is that the screening strategy recommended by the ATA is poorly selective, since almost $80 \%$ of our sample would be screened for thyroid dysfunction, according to the targeted high-risk case-finding approach. In Brazil, the occurrence of multiparity is quite frequent, especially among women of lower socio-economic levels. This is demonstrated in the present study, since 76 women $(25.7 \%)$ had previous history of $\geq 2$ prior pregnancies. If we disconsider multiparity as a positive criteria for thyroid screening, 221 women $(74.7 \%)$ would be classified as high risk, and 75 (25.3\%) would be classified as low risk for thyroid dysfunction during pregnancy. Thus, the inclusion of this new criteria in the ATA's most recent guidelines had little impact on the risk classification.

In our cohort, the prevalence of $\mathrm{OH}$ was $1.3 \%$ (n $=4$ ), which is slightly higher than that found in the literature (20). Regarding SCH, 17 patients (5.7\%) had TSH $>3.8 \mathrm{mIU} / \mathrm{L}$. The prevalence of subclinical thyroid disorder can vary widely, ranging from 2 to $35 \%$ of pregnancies, depending on the TSH reference values used to define $\mathrm{SCH}$ as well as by particularities of the population studied, such as iodine sufficiency and other demographic characteristics (20-22).

To our knowledge, this is the first study to evaluate the accuracy of the screening criteria proposed by the ATA in its most recent guidelines in the diagnosis of thyroid dysfunction, applying objective measurements of signs and symptoms of hypothyroidism, and in a population with known iodine sufficiency. All participants were included in their first trimester of gestation and had a comprehensive thyroid assessment including history, physical examination, and thyroid function tests.

For the first time, a validated clinical score was applied to assess signs and symptoms of thyroid dysfunction, which is one of the ATA's criteria for case finding. Although the Zulewski's clinical score has been validated for a non-pregnant population, Nazarpour and cols. showed in a recent study - published after the conclusion of our analyzes - that the Billewikz scoring system, which is an older version of the Zulewski's score, may be a reliable tool in the diagnosis of $\mathrm{OH}$ during pregnancy (23). It is important to highlight 2 limitations of this study. First, it is the relatively small sample size. Second, our cohort may not be 
representative of other populations with differences in ethnicity, iodine intake, and TSH reference values.

In conclusion, the screening criteria proposed by the ATA's 2017 guidelines were not accurate in the diagnosis of thyroid dysfunction in our cohort of pregnant women, since no significant association was found between risk classification and occurrence of SCH. Testing only the high-risk pregnant women in the targeted case-finding approach would miss around $40 \%$ of all hypothyroid patients.

Acknowledgements: this work was financially supported by Fundação Carlos Chagas Filho de Amparo à Pesquisa do Estado do Rio de Janeiro (FAPERJ) (grant number E-26/202.143/2015) in concept with Ministério da Saúde and Conselho Nacional de Pesquisa (CNPq).

Disclosure: no potential conflict of interest relevant to this article was reported.

\section{REFERENCES}

1. Abalovich M, Gutierrez S, Alcaraz G, Maccallini G, Garcia A, Levalle O. Overt and subclinical hypothyroidism complicating pregnancy. Thyroid. 2002;12(1):63-8.

2. Allan WC, Haddow JE, Palomaki GE, Williams JR, Mitchell ML, Hermos RJ, et al. Maternal thyroid deficiency and pregnancy complications: implications for population screening. J Med Screen. 2000;7(3):127-30.

3. Korevaar TI, Muetzel R, Medici M, Chaker L, Jaddoe VW, de Rijke $Y B$, et al. Association of maternal thyroid function during early pregnancy with offspring $\mathrm{IQ}$ and brain morphology in childhood: a population-based prospective cohort study. Lancet Diabetes Endocrinol. 2016;4(1):35-43.

4. Haddow JE, Palomaki GE, Allan WC, Williams JR, Knight GJ, Gagnon J, et al. Maternal thyroid deficiency during pregnancy and subsequent neuropsychological development of the child. $\mathrm{N}$ Engl J Med. 1999;341(8):549-55.

5. Alexander EK, Pearce EN, Brent GA, Brown RS, Chen H, Dosiou C, et al. 2017 Guidelines of the American Thyroid Association for the Diagnosis and Management of Thyroid Disease During Pregnancy and the Postpartum. Thyroid. 2017;27(3):315-89.

6. Lazarus J, Brown RS, Daumerie C, Hubalewska-Dydejczyk A, Negro R, Vaidya B. 2014 European thyroid association guidelines for the management of subclinical hypothyroidism in pregnancy and in children. EurThyroid J. 2014;3(2):76-94.

7. Vaidya B, Anthony S, Bilous M, Shields B, Drury J, Hutchison S, et al. Detection of thyroid dysfunction in early pregnancy: Universal screening or targeted high-risk case finding? J Clin Endocrinol Metab. 2007;92(1):203-7.

8. Chang DL, Leung AM, Braverman LE, Pearce EN. Thyroid testing during pregnancy at an academic Boston Area Medical Center. J Clin Endocrinol Metab. 2011;96(9):E1452-6.

9. Jouyandeh Z, Hasani-Ranjbar S, Qorbani M, Larijani B. Universal screening versus selective case-based screening for thyroid disorders in pregnancy. Endocrine. 2015;48(1):116-23.

10. Wilson JM, JungnerYG. [Principles and practice of mass screening for disease]. B Bol Oficina Sanit Panam. 1968;65(4):281-393.

11. Taylor PN ZS, MinT, Nagarahaj K, Lazarus J, Okosieme O. Thyroid Screening in Early Pregnancy: Pros and Cons. Front Endocrinol (Lausanne). 2018 Oct 25;9:626.

12. Saraiva DA, Morais N, Martins Corcino C, Martins Benvenuto Louro BerbaraT, Schtscherbyna A, Santos M, et al. lodine status of pregnant women from a coastal Brazilian state after the reduction in recommended iodine concentration in table salt according to governmental requirements. Nutrition. 2018;53:109-14.

13. Zulewski H, Muller B, Exer P, Miserez AR, Staub JJ. Estimation of tissue hypothyroidism by a new clinical score: evaluation of patients with various grades of hypothyroidism and controls. J Clin Endocrinol Metab. 1997;82(3):771-6.

14. Morais N, Assis ASA, Corcino CM, Saraiva DA, BerbaraT, Ventura $\mathrm{CDD}$, et al. Recent recommendations from ATA guidelines to define the upper reference range for serum TSH in the first trimester match reference ranges for pregnant women in Rio de Janeiro. Arch Endocrinol Metab. 2018;62(4):386-91.

15. Hope A. A simplified Monte Carlo significance test procedure. Journal of the Royal Statistical Society. 1968; Series B 30:582-98.

16. Šidàk Z. Rectangular confidence region for the means of multivariate normal distributions. J Am Stat Assoc. 1967;62:626-33.

17. Stagnaro-Green $A$, Abalovich $M$, Alexander E, Azizi F, Mestman $\mathrm{J}$, Negro R, et al. Guidelines of the American Thyroid Association for the diagnosis and management of thyroid disease during pregnancy and postpartum. Thyroid. 2011;21(10):1081-125.

18. Cohen J. Statistical power analysis for the behavioral sciences. 2nd ed. Hillsdale, N.J.: L. Erlbaum Associates; 1988. xxi, 567 p.

19. Rosario PW. Selective screening for thyroid dysfunction in pregnant women: How often do low-risk women cease to be treated following the new guidelines of the American Thyroid Association? Arch Endocrinol Metab. 2018;62(6):641-3.

20. Klein RZ, Haddow JE, Faix JD, Brown RS, Hermos RJ, Pulkkinen $A$, et al. Prevalence of thyroid deficiency in pregnant women. Clin Endocrinol (Oxf). 1991;35(1):41-6.

21. Kim HJ, ChoYY, Kim SW, KimTH, Jang HW, Lee SY, et al. Reference intervals of thyroid hormones during pregnancy in Korea, an iodine-replete area. Korean J Intern Med. 2018 May;33(3):552-60.

22. Mosso L, Martinez A, Rojas MP, Margozzini P, Solari S, Lyng $\mathrm{T}$, et al. [Frequency of subclinical thyroid problems among women during the first trimester of pregnancy]. Rev Med Chil. 2012;140(11):1401-8.

23. Nazarpour S, Ramezani Tehrani F, Rahmati M, Minooee S, Simbar M, Noroozzadeh M, et al. Validation of Billewicz Scoring System for Detection of Overt Hypothyroidism During Pregnancy. Int $J$ Endocrinol Metab. 2018;16(3):e64249. 